
Theoretical \& Applied Science

Dissertant, Azerbaijan Economic State University, Azerbaijan Republic

p-ISSN: 2308-4944 (print) e-ISSN: 2409-0085 (online) nauka-xxi@mail.ru

Year: 2017 Issue: 05 Volume: 49

Published: $30.05 .2017 \quad$ http://T-Science.org

SECTION 19. Management. Marketing. Public administration.

\title{
THE ISSUES OF AN OBJECTIVE ASSESSMENT OF COMMERCIAL RELATIONS IN THE CONSTRUCTION MATERIALS MARKET
}

Abstract: These issues of an objective assessment of commercial relations in the construction materials market are discussed in this article. The main features, criteria and principles of formation of commercial relations and mechanisms of commercial activity are analyzed. The scientific and practical views on the improvement of the main mechanisms of commercial relations in the construction materials market in the context of global economic challenges are formed. A number of proposals and recommendations on the issues under consideration are given.

Key words: commercial relations, commercial activities, objectivity, valuation, market of building materials.

Language: Russian

Citation: Fazail YI (2017) THE ISSUES OF AN OBJECTIVE ASSESSMENT OF COMMERCIAL RELATIONS IN THE CONSTRUCTION MATERIALS MARKET. ISJ Theoretical \& Applied Science, 05 (49): 217-221.

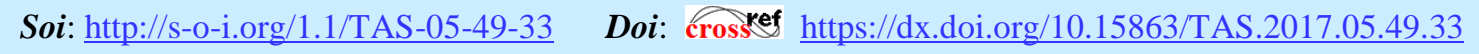

\section{ВОПРОСЫ ОБЪЕКТИВНОЙ ОЦЕНКИ КОММЕРЧЕСКИХ ОТНОШЕНИЙ НА РЫНКЕ СТРОИТЕЛЬНЫХ МАТЕРИАЛОВ}

Аннотация: В данной статье рассматриваются вопросы объективной оценки коммерческих отношений на рынке строительных материалов. Анализируются основные особенности, критерии и принщипь формирования коммерческих отношений и механизмов коммерческой деятельности. Сформированы научные и практичные взгляды совершенствования главных механизмов коммерческих отношений на рынке строительных материалов в контексте глобальных экономических вызовов. Дан ряд предложений и рекомендаций по рассматриваемым вопросам.

Ключевые слова: коммерческие отношения, коммерческая деятельность, объективность, оценка, рынок строительных материалов.

\section{Introduction}

В нынешних условиях вопросы объективной оценки коммерческих отношений на рынке строительных материалов обуславливают рассмотрение множественных проблем, изучение их сущности и особенностей, определение наиболее эффективных форм и путей коммерческих отношений с целью повышения рациональности и прибыльности коммерческой деятельности. С этой целью требуется особо обратить внимание на инклюзивность сферы производства строительных материалов, которая выступает в качестве важного начала звена по непрерывному обеспечению сотен наименований строительных материалов и изделий, конечных потребителей и тем самым способствует интенсификации деятельности коммерческих и строительных организаций, где формируются тесные коммерческие отношения. Более того, подобное развитие событий в данных сферах серьёзно влияет на развитие малого и среднего звена предпринимательского корпуса, способствует расширению бизнеса - среды, создает дополнительные возможности усиления занятости, создание новых рабочих мест, активизация трудового потенциала населения страны, продуктивного использования местных ресурсов, рабочий силы, запасов строительных материалов и прочих сырьевых компонентов.

\section{Materials and Methods}

Дело в том, что интенсификация деятельности строительного сектора, реализация крупных строительных проектов, в том числе 
огромной сети инфраструктурных объектов, здании, сооружении, спортивных комплексов, жилищных массивов, гражданских и промышленных строительных объектов нуждается в огромной массе и ассортименте строительных материалов и изделий. Поэтому для решения данной задачи между производителями и конечных потребителей строительных материалов и изделий рынок выступает в качестве одной из ведущих звеньев по организацию и развитию коммерческой деятельности, отвечающие по всем интересом участников рынка, где главным условиям является осуществление коммерческой деятельности с определенной прибылью. При этом необходимо интенсивно развивать существующие коммерческие отношения и торговые технологии, обеспечить прозрачные и взаимовыгодные коммерческие операции, объективную оценку в целом всей коммерческой деятельности на рынке строительных материалов. Для этого, в первую очередь требуется комплексное и системное изучение основных компонентов и элементов коммерческих отношений на рынке строительных материалов, оценить адекватность и эффективность существующих отношений с учётом интенсификации деятельности в целом строительного сектора и взаимодополняющих секторов национальной экономики в контексте активизации глобальных экономических вызовов и процессов трансформации традиционных хозяйственных и рыночных механизмов, практичных инструментов, подходов, систем управления и контроля и т.д.

Отметим, что эффективной деятельностью рынка строительных материалов требует рассмотрения и решения массовых проблем по активизации субъектов предпринимательской деятельности и бизнеса на данном рынке. Рынок строительных материалов должен базироваться на долгосрочных и взаимовыгодных коммерческих отношениях и узлах. Необходимо постоянно развивать материально-техническую базу рынка строительных материалов, создать мобильную и в то же время эффективную логистическую систему, структуру обеспечивающую соблюдение стандартов и законодательство в данном рынке и создать более благоприятные условия расширения участников рынка, притока финансовых вложений на увеличение объёма коммерческих операций, который позволил бы всем участникам рынка одновременно иметь положительный импульс развития и расширить свой бизнес, получить спрогнозированную прибыль. Исследователи Т. Мамедов и В. Тишенко подчёркивают важность разработки и осуществления новых механизмов по определению эффективности в целом строительного сектора, особенно в сферах производства строительного материала и рынка строительных материалов. При этом необходимо для объективного осуществления оценочной работы применять системный подход и больше опираться на научно-технические новшества и с учётом углубления рыночных и коммерческих отношений[1]. Если рассмотреть на примере рынка строительных материалов Азербайджана, то в первую очередь необходимо отметить потенциал расширения сети производства более конкурентоспособных строительных материалов и изделий за счёт освоения мощных залежей сырья для производства строительных материалов, особенно в регионах страны. Кроме того, необходимо объективно отнестись к объёму и размеру, грандиозным строительным работам в столице - в городе Баку, где используются качественные и довольно расширенные ассортименты строительных материалов и изделий, часть которых заводиться из зарубежа.

К сожалению, приходиться отметить, что существующий рынок строительных материалов и коммерческие отношения в нём отстают от адекватного уровня проводимых строительных работ в стране в целом. Одна из главных причин подобных ситуаций является недостаточное развитие коммерческих отношений и предпринимательства в данной отрасли. Правда, государство всеми способами старается и применяет государственные поддержки развития предпринимательства и повышения эффективности коммерческой деятельности на рынке строительных материалов, однако формирование и развитие более взаимовыгодных и продуктивных коммерческих отношений требует не малого времени и опыта в данной отрасли, который у азербайджанский компании и коммерческих субъектов недостаточно оценивается. Местным компаниям и коммерческим субъектам необходимо более глубоко освоить особенности и технологии сегментов и конъюнктур рынка строительных материалов, сформировать более доверительные и фундаментальные коммерческие отношения на долгосрочной основе и тщательно следить за происходящими событиями на данном рынке[2]. Более того, необходимо применить те методы и способы практичной деятельности, которые укрепляют существующие коммерческие отношения, повышают доверительные отношения между участниками рынка и между партнёрами, способствуют подготовке и реализации более продуктивных и выгодных коммерческих операций на рынке. Далее, очень важно оптимизировать расходы на организации и осуществление коммерческих операций и торговых технологий, тем самым обеспечить привлекательность подобных операций для всех 
участников рынка строительных материалов. Для этого, необходимо интенсивно применять инновационные методы и технологии, способствующие уменьшению цикла времени и основных расходов по организации и управлению потоков строительных материалов и изделий от производителей до конечных покупателей, согласно требованиям и спросам рынка строительных материалов. Профессиональный и сбалансированный коммерческие подходы и коммерческие отношения оказывают положительное влияние на продуктивность и рациональность на осуществляемые коммерческие деятельности. При этом считается важным рассмотрение более эффективных тенденций развития коммерческих отношений на рынке с учётом анализа сопоставления их положительных и отрицательных черт и осуществление объективной оценки с прогнозированием продвижения товара на рынке строительных материалов[3]. Известно, что продуктивного и интенсивного развития сферы производства строительных материалов и расширения рынка строительных материалов обуславливает постоянного притока инвестиционных ресурсов и требует адресного вложения инвестиционных ресурсов в данной сфере. Однако в условиях нарастания финансовых трудностей и ограничений кредитных ресурсов надеется на интенсивный рост инвестиционных вложений не приходиться. Поэтому часть этих проблем можно смягчить за счёт взаимопониманий коммерческих партнёров путем доверительных отношений, минимизации затрат за промежуточные коммерческие операции, оптимизацию прочих расходов, цен на строительных материалов и прочее. Более того, долгосрочные партнерские и коммерческие отношения могут способствовать принятию положительного решения на осуществление совместных инвестиций в промышленности строительных материалов и в том числе с участием иностранных партнёров и инвесторов[4]. Но для этого, реальный потенциал предприятий-производителей строительных материалов и коммерческих субъектов рынка строительных материалов должен подвергаться серьёзному изучению, уточнению с завершением объективной оценки потенциала того или иного участника строительного рынка на долгосрочной перспективе. При этом необходимые условия осуществления инвестиционной и коммерческой деятельности должны быть выгодными для всех участников коммерческих отношений с учётом оценки обеспечения требований конечных потребителей[5].

Отметим, что при осуществлении мероприятий связанных совершенствованием коммерческих отношений и механизмов коммерческой деятельности положительной основой считается объективная оценка существующих возможностей и резервов в деятельности коммерческих субъектов и участников рынка строительных материалов. Необходимо обратить внимание на адекватность существующих механизмов и компонентов, составляющих элементов коммерческих отношений, проблемы, разногласия, прочих факторов мешающих развитию в коммерческих отношений и применению более эффективных способов и методов расширения коммерческой деятельности на рынке строительных материалов[6;7;8]. Все эти вопросы и оценочные работы должны сопровождаться объективно, бесстрастно, на основе выбранных более эффективных методов и способов[9;10]. Кроме того, при оценке реальной положений и адекватности коммерческих отношений, необходимо придерживаться ряду критерий и принципов[11]. В Таблице 1 даны основные критерии и показатели, их сущности по оценке реального положения в коммерческих отношениях на рынке строительных материалов.

Таблица 1

Основные критерии, показатели, их сущность по оценке реального положения в коммерческих отношениях на рынке строительных материалов.

\begin{tabular}{|c|c|}
\hline Критерии и показатели & Краткое содержание \\
\hline $\begin{array}{l}\text { 1. Сбалансированный спрос и } \\
\text { предложение }\end{array}$ & $\begin{array}{l}\text { - } \\
\text { необходимо } \\
\text { предложение участников рынка строительных материалов; } \\
- \\
\text { рассмотрены и максимум обеспечены. }\end{array}$ \\
\hline $\begin{array}{l}2 . \\
\text { рыночной эконоюдение принципов } \\
\text { рынок }\end{array}$ & $\begin{array}{l}\text { - коммерческие } \\
\text { материалов должныения на } \\
\text { мыть сформированы }\end{array}$ \\
\hline
\end{tabular}




\section{Impact Factor:}

\begin{tabular}{|c|c|c|}
\hline ISRA (India) & $=1.344$ & SIS (USA) \\
\hline ISI (Dubai, UAE & 0.829 & РИНЦ $($ Russia $)=0.234$ \\
\hline tralia) & 0.564 & ESJI (KZ) \\
\hline & $=1.500$ & SJIF $($ Morocco $)=\mathbf{2}$ \\
\hline
\end{tabular}

$\begin{array}{ll}\text { ICV }(\text { Poland }) & =\mathbf{6 . 6 3 0} \\ \text { PIF } \text { (India) } & =\mathbf{1 . 9 4 0} \\ \text { IBI } \text { India) } & =\mathbf{4 . 2 6 0}\end{array}$

\begin{tabular}{|c|c|}
\hline $\begin{array}{l}3 . \quad \text { Соблюдение законности и } \\
\text { конкурентной среды }\end{array}$ & 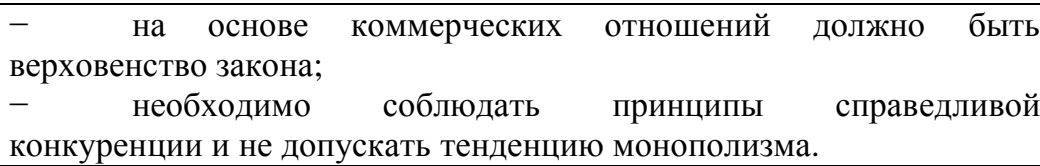 \\
\hline $\begin{array}{l}4 . \quad \text { Адекватность } \\
\text { инфраструктур и материально- } \\
\text { технической базы }\end{array}$ & 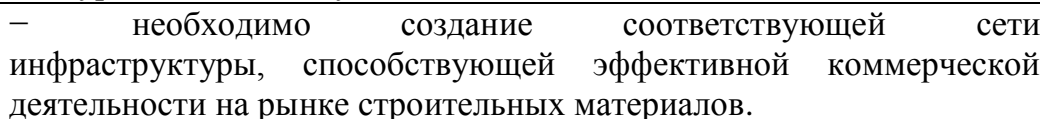 \\
\hline $\begin{array}{l}5 . \quad \text { Уровень выполнения } \\
\text { договорных обязательств }\end{array}$ & $\begin{array}{l}\text { - участники коммерческих отношений должны выполнять } \\
\text { взятие на себя договорных обязательств. } \\
\end{array}$ \\
\hline $\begin{array}{l}6 . \quad \text { Формирование } \\
\text { взаимовыгодных коммерческих } \\
\text { отношений }\end{array}$ & $\begin{array}{l}\text { - } \quad \text { формирование и развитие партнёрских отношений между } \\
\text { участниками рынка строительных материалов должны основываться } \\
\text { на принципах взаимной выгодности и прибыльности. }\end{array}$ \\
\hline $\begin{array}{l}7 . \quad \text { Рассмотрение проблемных } \\
\text { вопросов и принятие оптимальных } \\
\text { решений }\end{array}$ & 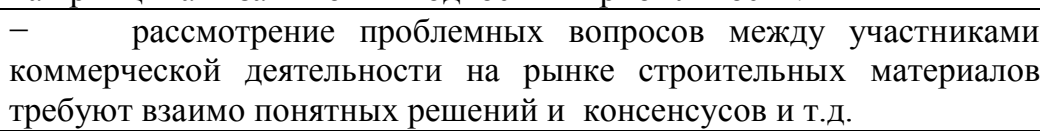 \\
\hline
\end{tabular}

Примечание: Таблица 1 разработана автором на основе исследовательских материалов.

Как видно из Таблицы 1 , в число основных критерий и показателей по оценке реального положения в коммерческих отношениях на рынке строительных материалов входят сбалансированность спроса и предложения участников рынка, соблюдение принципов рыночной экономики во всех циклах коммерческой деятельности, соблюдение законности и конкурентной среды между всеми участниками рынка строительных материалов, адекватность инфраструктур и материальнотехническая база рынка, уровень выполнения договорных обязательств участников коммерческих отношений и формирования взаимных выгодных коммерческих отношений, наконец, рассмотрение проблемных вопросов и принятие оптимальных решений на основе взаимопонятных решений и консенсуса.

\section{Conclusion}

Отметим, что материалы исследования позволяют сделать ряд выводов по вопросам объективной оценки коммерческих отношений на рынке строительных материалов:
- необходимо обеспечить оптимальные условия для развития коммерческих отношений на рынке строительных материалов с учётом интересов всех участников рынка и сторон коммерческой деятельности;

- одним из главных базовых принципов объективной оценки коммерческих отношений на рынке строительных материалов является объективная оценка реального положения и оптимизация взаимоотношений участников коммерческих операций с учётом спроса и предложения на рынке;

- необходимо учитывать все проблемные вопросы во взаимоотношениях участников рынка, раскрыть их порождающие причины с соответствующими выводами, построить долгосрочные взаимовыгодные коммерческие отношения между участниками рынка строительных материалов с учётом глобальных экономических вызовов и т.д.

\section{References:}

1. Mamedov T, Tishenko V (2017) Otsenka i podtverzhdeniye sootvetstviya $\mathrm{v}$ oblasti stroitel'stva. Available: http://www.fsrstroy.ru/archive/8013. (Accessed: 10.05.2017).

2. (2017) Rynok stroitel'nykh materialov. Mbagroup. Available: http://www.mbgroup.az/az/services/17. (Accessed: 10.05.2017).
3. Kotlyarova SN (2015) Metodika otsenki rynkov sbyta stroitel'nykh materialov//Problemy razvitiya territorii. Vypusk №1(75), -p. 63-75. Available: $\quad$ http://www.cyberleninka.ru. (Accessed: 10.05.2017).

4. Nabiyev RA (2008) Voprosy kompleksnoy otsenki effektivnosti sovmestnykh investitsiy $\mathrm{v}$ promyshlennost' stroitel'nykh materialov: na 


\begin{tabular}{l|lrl|l|ll} 
& ISRA (India) & $=\mathbf{1 . 3 4 4}$ & SIS (USA) & $=\mathbf{0 . 9 1 2}$ & ICV (Poland) & $=\mathbf{6 . 6 3 0}$ \\
Impact Factor: & ISI (Dubai, UAE) $=\mathbf{0 . 8 2 9}$ & PUHL (Russia) $=\mathbf{0 . 2 3 4}$ & PIF (India) & $=\mathbf{1 . 9 4 0}$ \\
& GIF (Australia) & $\mathbf{0 . 5 6 4}$ & ESJI (KZ) & $=3.860$ & IBI (India) & $=\mathbf{4 . 2 6 0}$ \\
& JIF & $=\mathbf{1 . 5 0 0}$ & SJIF (Morocco) & $=\mathbf{2 . 0 3 1}$ & & \\
\hline
\end{tabular}

primere Astrakhanskogo regiona. Diss. kand. ekon. nauk. Astrakhan', -166 p. Available: http://www.dslib.net (Accessed: 10.05.2017).

5. Lavrikova YG (2013) Razvitiye rynkov sbyta promyshlennoy produktsii Sverdlovskoy oblasti v usloviyakh VTO Tekst / YU.G. Lavrikova, S.N.Kotlyarova // Izvestiya Ural'skogo gosudarstvennogo gornogo universiteta. №4. p. 97-101.

6. (2017) Otsenka rynka stroitel'nykh uslug. Available: http://www.0zd.ru. (Accessed: 10.05.2017).

7. Kyazymly X (2008) Osnova otsenivaniya. Baku, izdatel'stvo «Universitet Ekonomiki», $430 \mathrm{p}$.

8. Kameneva SY (2017) Organizatsiya kommercheskoy deyatel'nosti v optovoy torgovle potrebitel'skoy kooperatsii i puti yeye sovershenstvovaniya: $\mathrm{Na}$ materialakh
Povolzhskogo regiona. Diss. kand. ekon. nauk, $235 \mathrm{p}$.

9. Teklin AB (2006) Marketingovyy analiz i prognoirovaniye urovnya, dinamiki i struktury optovykh tsen na stroitel'nyye materialy. Diss. kand. ekon. nauk. Moscow, $-177 \mathrm{p}$.

10. Serikov DA (2007) Konkurentnaya situatsiya na rynke proizvodstva stroitel'nykh materialov. Rol' i tendentsii razvitiya $\mathrm{v}$ ekonomike Krasnodarskogo kraya otrasli proizvodstva stroitel'nykh materialov. Kubanskiy Institut Mezhdunarodnogo Predprinimatel'stva i Mendzhmenta//Nauchnyy zhurnal KubGAU, №29(5).

11. Korostelev AA (2001) Otsenka ekonomicheskoy nadezhnosti stroitel'nykh organizatsiy. Diss. kand. ekon. nauk. Moscow, $-163 \mathrm{p}$. 